\title{
Gaussian null coordinates for rotating charged black holes and conserved charges
}

\author{
M. Cvetič ${ }^{1,2}$ C. N. Pope $\odot,{ }^{3,4}$ A. Saha $\odot,{ }^{3}$ and A. Satz ${ }^{5}$ \\ ${ }^{1}$ Department of Physics and Astronomy, University of Pennsylvania, \\ Philadelphia, Pennsylvania 19104, USA \\ ${ }^{2}$ Center for Applied Mathematics and Theoretical Physics, University of Maribor, \\ SI2000 Maribor, Slovenia \\ ${ }^{3}$ George P. \& Cynthia Woods Mitchell Institute for Fundamental Physics and Astronomy, \\ Texas A\&M University, College Station, Texas 77843, USA \\ ${ }^{4}$ DAMTP, Centre for Mathematical Sciences, Cambridge University, \\ Wilberforce Road, Cambridge CB3 OWA, United Kingdom \\ ${ }^{5}$ Sarah Lawrence College, Bronxville, New York 10708, USA
}

(Received 3 March 2020; accepted 9 March 2020; published 26 March 2020)

\begin{abstract}
Motivated by the study of conserved Aretakis charges for a scalar field on the horizon of an extremal black hole, we construct the metrics for certain classes of four-dimensional and five-dimensional extremal rotating black holes in Gaussian null coordinates. We obtain these as expansions in powers of the radial coordinate, up to sufficient order to be able to compute the Aretakis charges. The metrics we consider are for 4-charge black holes in four-dimensional STU supergravity $(N=2$ supergravity coupled to three vector multiplets) (including the Kerr-Newman black hole in the equal-charge case) and the general 3-charge black holes in five-dimensional STU supergravity. We also investigate the circumstances under which the Aretakis charges of an extremal black hole can be mapped by conformal inversion of the metric into NewmanPenrose charges at null infinity. We show that while this works for four-dimensional static black holes, a simple radial inversion fails in rotating cases because a necessary conformal symmetry of the massless scalar equation breaks down. We also discuss that a massless scalar field in dimensions higher than four does not have any conserved Newman-Penrose charge, even in a static asymptotically flat spacetime.
\end{abstract}

DOI: 10.1103/PhysRevD.101.066025

\section{INTRODUCTION}

In the last few years there have been many studies that have revealed that the horizon of an extremal black hole is unstable to small perturbations. These may be perturbations of the black hole metric itself, or perturbations of matter fields propagating in the black hole background. The simplest such examples arise by considering the perturbations of a scalar field [1-4]. Perturbations of other fields, including linearized gravity, were considered in [5]. The instabilities stem from the existence of conserved charges on the future horizon of the extremal black hole, which imply that physical perturbations do not decay at large values of the advanced time $v$. These conserved charges are known as Aretakis charges. They exist quite generally for any black hole with an extremal

Published by the American Physical Society under the terms of the Creative Commons Attribution 4.0 International license. Further distribution of this work must maintain attribution to the author(s) and the published article's title, journal citation, and DOI. Funded by SCOAP . horizon, but not for a nonextremal black hole with a bifurcate horizon.

General arguments for the existence of Aretakis charges in a black hole with an extremal horizon can be given, making use of the general near-horizon form of the metric for such black holes [6]. [The near-horizon metric is given in Eq. (2.1) below.] The metric in this form is written using Gaussian null coordinates (GNC). The Aretakis charges can be calculated explicitly for a given extremal black hole solution by casting the metric into the Gaussian null form. This is straightforward for a simple static example such as the extremal Reissner-Nordström solution, but it is rather less simple for a stationary metric such as the extremal Kerr solution. In such a case one cannot construct an exact expression for the metric in Gaussian null form, but fortunately it is sufficient to determine just the first few orders in a GNC expansion of the metric in powers of the radial coordinate measuring distance away from the horizon. This procedure was carried out for the Kerr metric in $[7,8]$. Essentially, the method used was to solve the equations for null geodesics, in an expansion in powers of the radial distance from the horizon. 
One of the main purposes of the present paper is to cast the metrics for certain classes of rotating extremal supergravity black holes into the Gaussian null form, thus allowing one to compute the conserved Aretakis charges in these spacetimes. Specifically, we carry out this procedure for the rotating extremal black holes in four-dimensional STU supergravity ( $N=2$ supergravity coupled to three vector multiplets) that carry four independent electric charges, and also for the general 3-charge rotating extremal black holes in five-dimensional STU supergravity. Specializations of these results encompass the previously derived expressions for the extremal Kerr metric in four dimensions [7] and the extremal Myers-Perry metric in five dimensions [8]. The intermediate stages in the calculations necessary for casting the supergravity black hole solutions into Gaussian null form are quite involved, but the final results that we obtain, at the order that is sufficient for calculating the Aretakis charges, are remarkably simple.

An intriguing observation, in the case of the extremal Reissner-Nordström (ERN) metric [9,10], is that by performing an inversion of the radial coordinate so that the horizon is mapped into future null infinity $\mathcal{I}^{+}$, and then extracting an overall conformal factor, the Aretakis charges on the null horizon of the ERN metric can be mapped into conserved Newman-Penrose charges at null infinity in the conformally inverted metric. In fact, this conformal inversion of the ERN metric actually maps it into the ERN metric again, a result that had been obtained many years previously by Couch and Torrence [11].

The mapping of Aretakis charges on an extremal horizon into Newman-Penrose charges on $\mathcal{I}^{+}$was investigated in a more general setting in [12]. It was shown that the conformal inversion of a general extremal black hole, written in Gaussian null coordinates, gives rise to a metric that was called weakly asymptotically flat (WAF) in [12]. This metric approaches Minkowski spacetime at infinity, but with rather weaker falloff conditions than those of an asymptotically flat spacetime written in Bondi-Sachs coordinates. It was shown in [12] that Newman-Penrose charges can be computed in the WAF spacetime obtained by conformal inversion of the original extremal black hole, and in various static examples the mapping between Aretakis and Newman-Penrose charges was exhibited. These generalized the mapping for extremal Reissner-Nordström that was found in $[9,10]$. In particular, in the more general examples, such as multicharge static extremal black holes in STU supergravity, the conformal inversion of the original metric does not give back the same metric again, unlike the ERN case.

The discussion in [12] in principle applied also to stationary extremal black holes that are not static. One can certainly again compute Newman-Penrose charges in the WAF metric obtained by conformal inversion. As we shall discuss in the present paper, however, in the stationary case there is a lacuna in the argument that would be needed in order to link the Newman-Penrose charges to the original Aretakis charges. Namely, in order to map the one into the other, it is necessary to be able to argue that the solutions of the massless scalar wave equation in the original black hole metric and in the conformally inverted WAF metric can be related by the necessary conformal transformation. This is fine as long as the Ricci scalar, which enters in the conformally invariant scalar equation $\left(\square-\frac{1}{6} R\right) \psi=0$, either is zero or else it goes to zero sufficiently rapidly in the asymptotic region. This is certainly true in the case of the extremal Reissner-Nordström solution, where $R$ vanishes, and also in the more complicated four-dimensional static supergravity black holes, where $R$ vanishes sufficiently rapidly asymptotically. But, as we show later, in the weakened falloff of the WAF metrics in the stationary case, the Ricci scalar does not fall off fast enough at infinity, and this provides an obstruction to being able to relate the Aretakis charges to the Newman-Penrose charges of the conformally inverted WAF metric, at least if we assume a simple inversion of the radial coordinate. In fact a manifestation of this problem was foreshadowed in the results in [13], where the Aretakis and Newman-Penrose charges were calculated in the case of the extremal Kerr black hole and its conformal inversion.

The focus in [12], concerning the relation between Aretakis charges and Newman-Penrose charges, was fourdimensional spacetimes. We also address in this paper the possible extension of these considerations to more than four dimensions. We show that conserved Aretakis charges for massless scalar fields exist in higher-dimensional extremal black holes also. However, as we show, Newman-Penrose charges for a massless scalar field no longer exist when one goes beyond four dimensions. This happens simply because there is a term in the large- $r$ expansion for the scalar equation written in Bondi-Sachs coordinates that presents an obstruction to the existence of conserved charges, and this term occurs with a dimension-dependent coefficient $(n-2)(n-4)$ that is absent in $n=4$ dimensions but not when $n \geq 5$.

\section{GENERAL FORMALISM}

The metric near the horizon of an extremal black hole in any dimension $n$ can always be written in Gaussian null coordinates, where it takes the form [6]

$$
\begin{aligned}
d s^{2}= & L(x)^{2}\left[-\rho^{2} F d v^{2}+2 d v d \rho\right] \\
& +\gamma_{I J}\left(d x^{I}-\rho h^{I} d v\right)\left(d x^{J}-\rho h^{J} d v\right),
\end{aligned}
$$

where $F, \gamma_{I J}$, and $h^{I}$ depend on the radial coordinate $\rho$ and on the coordinates $x^{I}$ on the (spherical) horizon, which is located at $\rho=0$. Near the horizon we may assume ${ }^{1}$

\footnotetext{
${ }^{1}$ In principle it would suffice to assume weaker asymptotic conditions on the metric functions as $\rho$ approaches zero (as discussed, for example, in [12]), but in practice these are the ones that arise in the black holes we shall be considering.
} 


$$
\begin{aligned}
F(\rho, x) & =1+\rho F_{1}(x)+\rho^{2} F_{2}(x)+\cdots, \\
\gamma_{I J}(\rho, x) & =\bar{\gamma}_{I J}(x)+\rho \gamma_{I J}^{(1)}(x)+\rho^{2} \gamma_{I J}^{(2)}(x)+\cdots, \\
h^{I}(\rho, x) & =h_{0}^{I}(x)+\rho h_{1}^{I}(x)+\rho^{2} h_{2}^{I}(x)+\cdots .
\end{aligned}
$$

We shall consider the Aretakis charges for a scalar field $\psi$ obeying the massless Klein-Gordon equation $\square \psi=0$. The solutions can be taken to have the small- $\rho$ expansion

$\psi(\rho, v, x)=\psi_{0}(v, x)+\rho \psi_{1}(v, x)+\rho^{2} \psi_{2}(v, x)+\cdots$.

From this, and the form of the metric expansion, it follows that on the horizon one has [12]

$$
\begin{gathered}
\frac{\partial}{\partial v}\left[2 \frac{\partial \psi}{\partial \rho}+\frac{1}{2} \psi \frac{\partial \log \gamma}{\partial \rho}\right]+h_{0}^{I} \partial_{I} \psi \\
+\frac{1}{\sqrt{\bar{\gamma}}} \partial_{I}\left(\sqrt{\bar{\gamma}} L^{2} \bar{\gamma}^{I J} \partial_{J} \psi\right)=0
\end{gathered}
$$

If this is integrated over the horizon, with measure $\sqrt{\gamma} d^{n-2} x$, the final term gives zero since it is a total derivative. The coordinates $x^{I}$ on the $(n-2)$-sphere horizon divide into azimuthal angular coordinates (like $\varphi$ on the 2-sphere), and latitude type coordinates (like $\theta$ on the 2 -sphere). The azimuthal coordinates are associated with Killing vectors. Crucially, for the extremal black hole metrics we shall be considering and as we shall see in detail later, Gaussian null coordinates can be chosen so that $h_{0}^{I}$ is zero for the index values $I$ corresponding to the latitude type coordinates. It follows that $h_{0}^{I} \partial_{I} \psi$ can be written as $(\sqrt{\bar{\gamma}})^{-1} \partial_{I}\left(\sqrt{\bar{\gamma}} h_{0}^{I} \psi\right)$, and thus this term is also a total derivative that integrates to zero. The upshot is that the quantity

$$
Q_{A}=\int \sqrt{\bar{\gamma}} d^{n-2} x\left[2 \frac{\partial \psi}{\partial \rho}+\frac{1}{2} \psi \frac{\partial \log \gamma}{\partial \rho}\right],
$$

known as the Aretakis charge, is conserved on the horizon, in the sense that $\partial_{v} Q_{A}=0$.

In the subsequent sections we shall calculate the Aretakis charge for the case of certain rotating charged extremal black holes in four-dimensional STU supergravity, and for the general 3-charge extremal rotating black holes in fivedimensional STU supergravity. The key part of the calculations involves constructing the expressions for the black hole solutions in Gaussian null coordinates, up to the necessary order in the expansion in powers of $\rho$.

A technique for constructing Gaussian null coordinates for an extremal black hole metric has been described in $[7,8]$. Essentially, one writes down the equations for null geodesics in the extremal metric, for which first integrals exist for the time and the azimuthal coordinate(s). The equations for the remaining coordinates cannot be integrated explicitly, so one then expands these in power series in the affine parameter $\lambda$ along the geodesics. The geodesic equations are then integrated order by order in $\lambda$, imposing certain transversality conditions in the process. Finally, a change of variable from $\lambda$ to $\rho$ brings the metric into the desired form (2.1).

\section{EXTREMAL ROTATING STU BLACK HOLES IN FOUR DIMENSIONS}

\section{A. 4-charge STU black holes}

The 4-charge four-dimensional STU supergravity black holes that we shall be considering here were constructed in [14]. A convenient presentation for our purposes can be found in [15]. The metric can be written as

$$
\begin{aligned}
d s^{2}= & -\frac{\bar{\rho}^{2}-2 m r}{W}\left(d t+\mathcal{B}_{(1)}\right)^{2} \\
& +W\left(\frac{d r^{2}}{\Delta}+d \theta^{2}+\frac{\Delta \sin ^{2} \theta d \tilde{\phi}^{2}}{\bar{\rho}^{2}-2 m r}\right), \\
\mathcal{B}_{(1)}= & \frac{2 m a \sin ^{2} \theta\left(r \Pi_{c}-(r-2 m) \Pi_{s}\right)}{\bar{\rho}^{2}-2 m r} d \tilde{\phi}, \\
\bar{\rho}^{2}= & r^{2}+a^{2} \cos ^{2} \theta, \quad \Delta=r^{2}-2 m r+a^{2}, \\
W^{2}= & R_{1} R_{2} R_{3} R_{4}+a^{4} \cos ^{4} \theta \\
& +\left[2 r^{2}+2 m r \sum_{i} s_{i}^{2}+8 m^{2}\left(\Pi_{c}-\Pi_{s}\right) \Pi_{s}\right. \\
& \left.-4 m^{2}\left(s_{1}^{2} s_{2}^{2} s_{3}^{2}+s_{1}^{2} s_{2}^{2} s_{4}^{2}+s_{1}^{2} s_{3}^{2} s_{4}^{2}+s_{2}^{2} s_{3}^{2} s_{4}^{2}\right)\right] \\
& \times a^{2} \cos ^{2} \theta, \quad \Pi_{c}=\prod_{i} c_{i}, \quad \Pi_{s}=\prod_{i} s_{i}, \\
R_{i}= & r+2 m s_{i}^{2}, \quad \Pi_{i}
\end{aligned}
$$

where $s_{i}=\sinh \delta_{i}$ and $c_{i}=\cosh \delta_{i}$. The physical mass $M$ and the four physical charges $Q_{i}$ are given by ${ }^{2}$

$M=m+\frac{1}{2} m \sum_{i} s_{i}^{2}, \quad Q_{i}=2 m s_{i} c_{i}=m \sinh 2 \delta_{i}$.

The metric is extremal when $m=a$, which we assume from now on. This implies $\Delta=(r-a)^{2}$. Defining a new azimuthal coordinate

$$
\phi=\tilde{\phi}-\frac{1}{2 a\left(\Pi_{c}+\Pi_{s}\right)} t
$$

so that $\partial / \partial t$ is the Killing vector that becomes null on the horizon, we may write the extremal metric in the form

\footnotetext{
${ }^{2}$ In the case where the charges are set equal, with $\delta_{i}=\delta$, the solution reduces to the Kerr-Newman black hole, with $\bar{r}=r+$ $2 m \sinh ^{2} \delta$ being the standard Kerr-Newman radial coordinate. We shall discuss the extremal Kerr-Newman metric in Gaussian null coordinates in the next subsection.
} 


$$
\begin{aligned}
d s^{2}= & W^{-1}\left(A d t^{2}+2 B d t d \phi+C d \phi^{2}\right) \\
& +W\left[\frac{d r^{2}}{(r-a)^{2}}+\frac{d u^{2}}{1-u^{2}}\right]
\end{aligned}
$$

where $u=\cos \theta$.

In algebraic computations the parametrization of the charges in terms of the four parameters $s_{i}$ is not ideal, since the relation to the $c_{i}$ involves square roots, namely $c_{i}=\sqrt{1+s_{i}^{2}}$. We have found it more convenient here to work instead with the five parameters $\left(\alpha, \beta, \gamma, \Pi_{c}, \Pi_{s}\right)$, where

$$
\begin{aligned}
\alpha & =s_{1}^{2}+s_{2}^{2}+s_{3}^{2}+s_{4}^{2}, \\
\beta & =s_{1}^{2} s_{2}^{2}+s_{1}^{2} s_{3}^{2}+s_{1}^{2} s_{4}^{2}+s_{2}^{2} s_{3}^{2}+s_{2}^{2} s_{4}^{2}+s_{3}^{2} s_{4}^{2}, \\
\gamma & =s_{1}^{2} s_{2}^{2} s_{3}^{2}+s_{1}^{2} s_{2}^{2} s_{4}^{2}+s_{1}^{2} s_{3}^{2} s_{4}^{2}+s_{2}^{2} s_{3}^{2} s_{4}^{2}, \\
\Pi_{c} & =c_{1} c_{2} c_{3} c_{4}, \quad \Pi_{s}=s_{1} s_{2} s_{3} s_{4} .
\end{aligned}
$$

These are related by the identity

$$
\gamma=\Pi_{c}^{2}-\Pi_{s}^{2}-1-\alpha-\beta,
$$

and in practice we find it most convenient to use $\left(\alpha, \beta, \Pi_{c}, \Pi_{s}\right)$ as the four independent quantities that parametrize the four charges. In terms of $\left(\alpha, \beta, \gamma, \Pi_{c}, \Pi_{s}\right)$ the charge-dependent quantities $W$ and $\mathcal{B}_{(1)}$ in the metric (3.1), subject to the extremality condition $m=a$, are given by

$$
\begin{aligned}
W^{2}= & r^{4}+2 a \alpha r^{3}+2 a^{2}\left(2 \beta+x^{2}\right) r^{2}+2 a^{3}\left(4 \gamma+x^{2}\right) r \\
& +a^{4}\left[16 \Pi_{s}^{2}+4\left(2 \Pi_{c} \Pi_{s}-2 \Pi_{s}^{2}-\gamma\right) u^{2}+u^{4}\right], \\
\mathcal{B}_{(1)}= & \frac{2 a^{2} \sin ^{2} \theta\left(r \Pi_{c}-(r-2 a) \Pi_{s}\right)}{\bar{\rho}^{2}-2 a r} d \tilde{\phi} .
\end{aligned}
$$

Solving now for the null geodesics in this geometry, the first integrals for the ignorable coordinates $t$ and $\phi$ are taken to be

$$
\dot{t}=-\frac{C}{W(r-a)^{2}\left(1-u^{2}\right)}, \quad \dot{\phi}=\frac{B}{W(r-a)^{2}\left(1-u^{2}\right)},
$$

where a dot denotes a derivative with respect to the affine parameter $\lambda$. We now expand the $r$ and $u$ coordinates as power series in $\lambda$, with

$$
r=a+\sum_{n \geq 1} R_{n}(y) \lambda^{n}, \quad u=y+\sum_{n \geq 2} X_{n}(y) \lambda^{n},
$$

where the affine parameter $\lambda$ vanishes on the horizon. Substituting these expansions in to null geodesic constraint $g_{\mu \nu} \dot{x}^{\mu} \dot{x}^{\nu}=0$ and the Euler-Lagrange equation for $u$ allows us to solve iteratively for the $R_{n}(y)$ and $X_{n}(y)$ coefficients in (3.9). We find

$$
\begin{aligned}
& R_{1}(y)=\frac{2\left(\Pi_{c}+\Pi_{s}\right)}{s(y)}, \quad X_{2}(y)=\frac{y\left(1-y^{2}\right)}{2 a^{2} s(y)^{2}}, \\
& R_{2}(y)=\frac{2\left(\Pi_{c}+\Pi_{s}\right)\left(1-y^{2}\right)\left[4\left(\Pi_{c}-\Pi_{s}\right)^{3}+(9+7 \alpha+4 \beta) \Pi_{s}-(5+5 \alpha+4 \beta) \Pi_{c}-\left(\Pi_{c}-\Pi_{s}\right) y^{2}\right]}{a s(y)^{4}},
\end{aligned}
$$

where

$$
\begin{aligned}
s(y)^{2} & =1+2 \alpha+4 \beta+8 \gamma+16 \Pi_{s}^{2}+2\left(1+\alpha-2 \gamma+4 \Pi_{c} \Pi_{s}-4 \Pi_{s}^{2}\right) y^{2}+y^{4}, \\
& =\prod_{i}\left(1+2 s_{i}^{2}\right)+2\left(1+\sum_{i} s_{i}^{2}-2 \sum_{i<j<k} s_{i}^{2} s_{j}^{2} s_{k}^{2}+4 \Pi_{c} \Pi_{s}-4 \Pi_{s}^{2}\right) y^{2}+y^{4} .
\end{aligned}
$$

To the order in $\lambda$ we are working, the coefficient $R_{3}(y)$ is needed also. We shall not present this here, since it is rather complicated.

The expansions (3.9) can now be used in (3.8) yielding, after integration with respect to $\lambda$,

$t=v+T(y, \lambda)+f_{t}(y), \quad \phi=\chi+\Phi(y, \lambda)+f_{\phi}(y)$.

Here $T(y, \lambda)$ and $\Phi(y, \lambda)$ are the "naive" integrals of (3.8) with respect to $\lambda$. The functions of integration $f_{t}(y)$ and $f_{\phi}(y)$ are determined by requiring $V \cdot \partial_{y}=0$, and $\left(\partial_{y} \cdot \partial_{\chi}\right)_{\lambda=0}=0$. Substituting (3.9) and (3.12) into the metric (3.4), and working up to and including linear order in $\lambda$, we obtain the extremal 4-charge metrics in Gaussian null coordinates $(v, \lambda, y, \chi){ }^{3}$

\footnotetext{
${ }^{3}$ Knowing just the expansion coefficients $R_{1}, R_{2}, R_{3}$, and $X_{2}$ in (3.9) is sufficient to calculate all the metric components up to and including linear order in $\lambda$, except a term involving $\lambda d \lambda^{2}$. Showing that this term is actually zero would require knowing also the coefficients $R_{4}$ and $X_{3}$, in the procedure we have just described. However, a simple argument shows that this term is actually absent, since $g_{\lambda \lambda}=\dot{x}^{\mu} \dot{x}^{\nu} g_{\mu \nu}$, which vanishes by the null geodesic constraint.
} 
Finally, we make a further coordinate transformation from $\lambda$ to $\rho$, of the form

$$
\lambda=w(y) \rho .
$$

The function $w(y)$ is determined by the requirement that the metric be expressible in the form (2.1) with, in particular, the function $F\left(\rho, x^{I}\right)$ being equal to 1 at $\rho=0$. This gives a first-order nonlinear differential equation for $w$. If we define a new function $u(y)$ such that $w(y)=a^{2} s(y)\left[\frac{1}{2}-u(y)\right]$ then we find that $u$ must satisfy

$$
\left(1-y^{2}\right) u^{\prime 2}+u^{2}-\frac{1}{4}=0,
$$

and so in terms of a new angular coordinate $\vartheta$ such that $y=\cos \vartheta$, the solutions for $u$ are

$u=\frac{1}{2}, \quad$ or $\quad u=-\frac{1}{2}, \quad$ or $\quad u=\frac{1}{2} \sin (\vartheta+k)$,

where $k$ is an arbitrary constant of integration. The metric functions $L(x)$ and $h_{0}^{I}$ in the metric (2.1) are then given by

$$
\begin{aligned}
& L^{2}=w(y), \quad h_{0}^{y}=\left(1-y^{2}\right) u^{\prime}(y), \\
& h_{0}^{\chi}=-\frac{\left(\Pi_{c}-\Pi_{s}\right)}{\left(\Pi_{c}+\Pi_{s}\right)}\left[\frac{1}{2}-u(y)\right] .
\end{aligned}
$$

In order for the metric (2.1) to be nondegenerate $L(x)^{2}$, and hence $w(y)$, should be positive everywhere on the sphere. The first solution in (3.15) is trivial, implying $w(y)=0$ and hence $L=0$, so this is excluded. The second solution gives

$w(y)=a^{2} s(y), \quad h_{0}^{y}=0, \quad h_{0}^{\chi}=-\frac{\left(\Pi_{c}-\Pi_{s}\right)}{\left(\Pi_{c}+\Pi_{s}\right)}$,

and so $w(y)$ is indeed positive everywhere on the sphere. Note that $h_{0}^{y}=0$ for this solution. As we noted previously when discussing the derivation of the Aretakis charge, it is necessary that $h_{0}^{y}$ vanish in order for the charge to be conserved on the horizon. The third solution in (3.15) gives

$$
\begin{aligned}
w(y) & =\frac{1}{2} a^{2} s(y)[1-\sin (\vartheta+k)], \\
h_{0}^{y} & =-\sin \vartheta \cos (\vartheta+k), \\
h_{0}^{\chi} & =-\frac{\left(\Pi_{c}-\Pi_{s}\right)}{2\left(\Pi_{c}+\Pi_{s}\right)}[1-\sin (\vartheta+k)] .
\end{aligned}
$$

The function $w(y)$ will be positive everywhere on the sphere provided that $k$ is chosen appropriately. However, $h_{0}^{y}$ is nonzero, and so there will be no conserved Aretakis charge in this case. Thus we are led to choose the second solution in (3.15), implying that

$$
\lambda=a^{2} s(y) \rho
$$

where $s(y)$ is given in (3.11). The metric then takes the form (2.1) with

$L(y)^{2}=a^{2} s(y), \quad F=1-2 a\left(\Pi_{c}-\Pi_{s}\right) \rho+\cdots$,

and

$$
\begin{aligned}
h_{0}^{y}= & 0, \quad h_{0}^{\chi}=-\frac{\left(\Pi_{c}-\Pi_{s}\right)}{\Pi_{c}+\Pi_{s}}, \\
\bar{\gamma}_{y y}= & \frac{a^{2} s(y)}{1-y^{2}}, \quad \bar{\gamma}_{x \chi}=\frac{4 a^{2}\left(\Pi_{c}+\Pi_{s}\right)^{2}\left(1-y^{2}\right)}{s(y)}, \quad \bar{\gamma}_{y \chi}=0, \\
\gamma_{y y}^{(1)}= & \frac{2 a^{3}\left(\Pi_{c}+\Pi_{s}\right)\left[4 \Pi_{c}^{2}-4 \Pi_{s}^{2}-(2+\alpha)\left(1-y^{2}\right)\right]}{\left(1-y^{2}\right) s(y)}, \\
\gamma_{y x}^{(1)}= & \frac{4 a^{3}\left(\Pi_{c}+\Pi_{s}\right) y\left(1-y^{2}\right)}{s(y)}, \\
\gamma_{x x}^{(1)}= & \frac{8 a^{3}\left(\Pi_{c}+\Pi_{s}\right)^{2}\left(1-y^{2}\right)}{s(y)^{4}}\left[( \Pi _ { c } + \Pi _ { s } ) \left((2+\alpha)\left(1-y^{2}\right)\right.\right. \\
& \left.\left.-4 \Pi_{c}^{2}+4 \Pi_{s}^{2}\right)+2\left(\Pi_{c}-\Pi_{s}\right) s(y)\right],
\end{aligned}
$$

We have calculated $h_{1}^{y}$ and $h_{1}^{\chi}$ but they are rather complicated, and we shall not present them here since they are not required for our subsequent purposes.

It is now a straightforward matter to calculate the Aretakis charge (2.5) for the scalar field in this extremal black hole. From (3.21) the evaluation of $\partial \log \gamma / \partial \rho$ on the horizon gives

$$
\left.\frac{\partial \log \gamma}{\partial \rho}\right|_{\rho=0}=4 a\left(\Pi_{c}-\Pi_{s}\right)
$$

and hence the Aretakis charge (2.5) is given by

$$
Q_{A}=2 a^{2}\left(\Pi_{c}+\Pi_{s}\right) \int d y d \chi\left[2 \frac{\partial \psi}{\partial \rho}+2 a\left(\Pi_{c}-\Pi_{s}\right) \psi\right]
$$

evaluated at $\rho=0$, i.e.,

$$
Q_{A}=2 a^{2}\left(\Pi_{c}+\Pi_{s}\right) \int d y d \chi\left[2 \psi_{1}+2 a\left(\Pi_{c}-\Pi_{s}\right) \psi_{0}\right] .
$$




\section{B. Kerr-Newman black holes}

In the case where the four electric charges in the STU black hole are set equal, by taking $\delta_{i}=\delta$, and hence $s_{i}=s$, $c_{i}=c$, one obtains the Kerr-Newman black hole. From (3.2) the physical mass $M$ and electric charge $Q=Q_{i}$ become

$$
M=m\left(1+2 s^{2}\right), \quad Q=2 m s c,
$$

and so the extremality condition $a=m$ can be written as

$$
M^{2}=a^{2}+Q^{2}
$$

The function $s(y)$ in (3.11) is now polynomial in $y$, given by

$$
a^{2} s(y)=M^{2}+a^{2} y^{2}
$$

and the metric coefficients in (3.20) and (3.21) become

$$
L(y)^{2}=M^{2}+a^{2} y^{2}, \quad F=1-2 M \rho+\cdots,
$$

and

$$
\begin{aligned}
& h_{0}^{y}=0, \quad h_{1}^{y}=-\frac{2 a^{2} M\left(a^{2}+M^{2}\right) y\left(1-y^{2}\right)}{\left(M^{2}+a^{2} y^{2}\right)^{2}}, \\
& h_{0}^{\chi}=-\frac{2 a M}{a^{2}+M^{2}}, \quad h_{1}^{\chi}=\frac{a\left[2 M^{4}+a^{2} M^{2}\left(1-y^{2}\right)-a^{4} y^{2}\left(1+y^{2}\right)\right]}{\left(a^{2}+M^{2}\right)\left(M^{2}+a^{2} y^{2}\right)}, \\
& \gamma_{y y}^{(0)}=\frac{M^{2}+a^{2} y^{2}}{1-y^{2}}, \quad \gamma_{x \chi}^{(0)}=\frac{\left(a^{2}+M^{2}\right)^{2}\left(1-y^{2}\right)}{M^{2}+a^{2} y^{2}}, \quad \gamma_{y x}^{(0)}=0, \\
& \gamma_{y y}^{(1)}=\frac{2 M\left(a^{2}+M^{2}\right)}{\left(1-y^{2}\right)}, \quad \gamma_{x x}^{(1)}=\frac{2 M\left(a^{2}+M^{2}\right)^{2}\left(1-y^{2}\right)\left[M^{2}-a^{2}\left(1-2 y^{2}\right)\right]}{\left(M^{2}+a^{2} y^{2}\right)^{2}},
\end{aligned}
$$

$$
\gamma_{y x}^{(1)}=\frac{2 a^{3}\left(a^{2}+M^{2}\right) y\left(1-y^{2}\right)}{\left(M^{2}+a^{2} y^{2}\right)} .
$$

The Aretakis charge (3.24) becomes

$$
Q_{A}=\left(a^{2}+M^{2}\right) \int d y d \chi\left(2 \psi_{1}+M \psi_{0}\right)
$$

It is worth remarking that in the case of pairwise-equal charges, where we take, for example, $\delta_{3}=\delta_{1}$ and $\delta_{4}=\delta_{2}$, the function $s(y)$ given in (3.11) again becomes purely polynomial in $y$, with

$$
s(y)=\left(1+2 s_{1}^{2}\right)\left(1+2 s_{2}^{2}\right)+y^{2},
$$

and, as can straightforwardly be seen, the expressions for the metric coefficients in (3.20) and (3.21) again simplify considerably. The extremality condition $a=m$, expressed in terms of the physical mass and charges, is now

$$
a^{2}=\frac{\left(2 M-Q_{1}-Q_{2}\right)\left(2 M-Q_{1}+Q_{2}\right)\left(2 M+Q_{1}-Q_{2}\right)\left(2 M+Q_{1}+Q_{2}\right)}{16 M^{2}} .
$$

\section{EXTREMAL ROTATING STU BLACK HOLES IN FIVE DIMENSIONS}

The metric for the general 3-charge rotating black holes of five-dimensional STU supergravity was constructed in [16]. It is convenient to introduce the quantities $\alpha, \beta, \Pi_{s}$, and $\Pi_{c}$, defined in terms of the boost parameters $\delta_{i}$ for $i=1,2$, and 3 by

$$
\begin{aligned}
\alpha & =s_{1}^{2}+s_{2}^{2}+s_{3}^{2}, \quad \beta=s_{1}^{2} s_{2}^{2}+s_{1}^{2} s_{3}^{2}+s_{2}^{2} s_{3}^{2}, \\
\Pi_{s} & =s_{1} s_{2} s_{3}, \quad \Pi_{c}=c_{1} c_{2} c_{3},
\end{aligned}
$$

where $s_{i}=\sinh \delta_{i}$ and $c_{i}=\cosh \delta_{i}$. They obey the relation

$$
\Pi_{c}^{2}=1+\alpha+\beta+\Pi_{s}^{2},
$$

which we employ in order to eliminate $\beta$. We use the coordinates $t, r, \theta, \tilde{\phi}$, and $\tilde{\psi}$ of [16], with the redefinition $u=\cos \theta$. (We have put tildes on the two azimuthal angles because we will shortly redefine untilded versions with respect to which null vector on the horizon is simply $\partial / \partial t$.) Extremality of the metric is achieved by taking mass parameter $\mu=(a+b)^{2}$, where $a$ and $b$ are the two rotation parameters (called $l_{1}$ and $l_{2}$ in [16]); the double horizon is then at $r=\sqrt{a b}$. The metric can be written as 


$$
\begin{aligned}
d s^{2}= & \Delta^{-2 / 3}\left(A d t^{2}+2 B_{1} d t d \phi+2 B_{2} d t d \psi\right. \\
& \left.+C_{1} d \phi^{2}+C_{2} d \psi^{2}+2 C_{12} d \phi d \psi\right) \\
& +\Delta^{1 / 3}\left[\frac{d r^{2}}{\left(r^{2}-a b\right)^{2}}+\frac{d u^{2}}{1-u^{2}}\right]
\end{aligned}
$$

where

$$
\Delta=(a+b)^{6} \Pi_{s}^{2}+(a+b)^{4} \beta \rho^{2}+(a+b)^{2} \alpha \rho^{4}+\rho^{6},
$$

and $\rho^{2}=r^{2}+a^{2} u^{2}+b^{2}\left(1-u^{2}\right)$. The coordinate redefinitions for the untilded azimuthal coordinates are

$$
\begin{aligned}
& \phi=\tilde{\phi}-\frac{1}{(a+b)\left(\Pi_{c}+\Pi_{s}\right)} t, \\
& \psi=\tilde{\psi}-\frac{1}{(a+b)\left(\Pi_{c}+\Pi_{s}\right)} t .
\end{aligned}
$$

The functions $A, B_{1}, B_{2}, C_{1}, C_{2}$, and $C_{12}$ can be read off by starting from the metric given in Eq. (18) of [16] and applying the steps detailed above.

We proceed along the same lines as in the previous fourdimensional examples, with the appropriate generalizations to five dimensions. The case of the uncharged five-dimensional Myers-Perry black hole is discussed in detail in [8]. The first integrals for the geodesics in the $t, \phi$, and $\psi$ directions are chosen so that

$$
\begin{aligned}
A \dot{t}+B_{1} \dot{\phi}+B_{2} \dot{\psi} & =\Delta^{2 / 3}, \\
B_{1} \dot{t}+C_{1} \dot{\phi}+C_{12} \dot{\psi} & =0, \\
B_{2} \dot{t}+C_{2} \dot{\psi}+C_{12} \dot{\phi} & =0 .
\end{aligned}
$$

We then make the expansions

$$
r^{2}=a b+\sum_{n \geq 1} R_{n}(y) \lambda^{n}, \quad u=y+\sum_{n \geq 2} X_{n}(y) \lambda^{n},
$$

where $\lambda$ is the affine parameter along the null geodesic. Plugging these into the null constraint equation $L \equiv$ $\frac{1}{2} g_{\mu \nu} \dot{x}^{\mu} \dot{x}^{\nu}=0$ and the second-order Euler-Lagrange equation for $u$ allows us to solve iteratively for the $R_{n}(y)$ and $X_{n}(y)$ coefficient functions. We find

$$
\begin{aligned}
& R_{1}(y)=\frac{2 \sqrt{a b}(a+b)\left(\Pi_{c}+\Pi_{s}\right)}{s(y)}, \quad X_{2}(y)=\frac{(a-b) y\left(1-y^{2}\right)}{2(a+b) s(y)^{2}}, \\
& R_{2}(y)=\frac{(a+b)\left(\Pi_{c}+\Pi_{s}\right)}{3 s(y)^{5}}\left[3(a+b)\left(\Pi_{c}-\Pi_{s}\right) s(y)^{3}-2 a b\left(\Pi_{c}+\Pi_{s}\right) F_{1}\right],
\end{aligned}
$$

where $s(y)$ is given by

$$
s(y)=\prod_{i=1}^{3}\left[a y^{2}+b\left(1-y^{2}\right)+(a+b) s_{i}^{2}\right]^{1 / 3},
$$

and $F_{1}$ is given in (4.15) below. To the order in $\lambda$ that we are working, we also need $R_{3}(y)$. We have calculated this but it is too complicated to present explicitly here. Substituting these results into Eq. (4.6) enables us to solve for $\dot{t}, \dot{\phi}$, and $\dot{\psi}$. After integration, we have

$$
\begin{aligned}
t & =v+T(y, \lambda)+f_{t}(y), \quad \phi=\chi+\Phi(y, \lambda)+f_{\phi}(y), \\
\psi & =\sigma+\Psi(y, \lambda)+f_{\psi}(y),
\end{aligned}
$$

where $T, \Phi$, and $\Psi$ are the "naive" $\lambda$ integrals, as discussed in the four-dimensional examples previously. The functions of integration $f_{t}(y), f_{\phi}(y)$, and $f_{\psi}(y)$ are determined by requiring $V \cdot \partial_{y}=0$, where $V=\dot{x}^{\mu} \partial_{\mu}$, along with $\left(\partial_{y} \cdot \partial_{\chi}\right)_{\lambda=0}=0$ and $\left(\partial_{y} \cdot \partial_{\sigma}\right)_{\lambda=0}=0$.

With these preliminaries, we now have the necessary coordinate redefinitions to re-express the metric in terms of the Gaussian null coordinates $(v, \lambda, y, \chi, \sigma)$. A coordinate transformation

$$
\lambda=(a+b) s(y)\left[\frac{1}{8}-u(y)\right] \rho
$$

then casts it into the form (2.1), with $F\left(\rho, x^{I}\right)=1$ at $\rho=0$, provided that $u(y)$ obeys

$$
y(1-y) u^{\prime 2}+u^{2}-\frac{1}{64}=0 .
$$

Letting $y=\frac{1}{2}(1+\cos \vartheta)$, this implies

$u=\frac{1}{8}, \quad$ or $\quad u=-\frac{1}{8}, \quad$ or $\quad u=\frac{1}{8} \sin (\vartheta+k)$,

where $k$ is an arbitrary constant. The metric function $h_{y}^{0}$ is given by $h_{y}^{0}=4 y(1-y) d u / d y$, and thus we have just the one solution $u=-\frac{1}{8}$ that gives both a nonsingular coordinate transformation (4.11) and a vanishing $h_{y}^{0}$ (as is required for obtaining an Aretakis charge). The final form of the five-dimensional 3-charge extremal STU black holes in Gaussian null coordinates is then given by (2.1) with 


$$
\begin{aligned}
L(y)^{2} & =\frac{1}{4}(a+b) s(y) \quad F=1-\frac{(a+b)^{2}\left(\Pi_{c}-\Pi_{s}\right)}{4 \sqrt{a b}} \rho+\cdots, \\
h_{0}^{y} & =0, \quad h_{0}^{\chi}=-\frac{\left(b \Pi_{c}-a \Pi_{s}\right)}{2 \sqrt{a b}\left(\Pi_{c}+\Pi_{s}\right)}, \quad h_{0}^{\sigma}=-\frac{\left(a \Pi_{c}-b \Pi_{s}\right)}{2 \sqrt{a b}\left(\Pi_{c}+\Pi_{s}\right)}, \\
\bar{\gamma}_{y y} & =\frac{(a+b) s(y)}{1-y^{2}}, \quad \bar{\gamma}_{\chi \chi}=\frac{(a+b) H_{2}\left(1-y^{2}\right)}{s(y)^{2}}, \quad \bar{\gamma}_{\sigma \sigma}=\frac{(a+b) \tilde{H}_{2} y^{2}}{s(y)^{2}}, \\
\bar{\gamma}_{\chi \sigma} & =\frac{(a+b) H_{0} y^{2}\left(1-y^{2}\right)}{s(y)^{2}}, \quad \gamma_{y y}^{(1)}=\frac{\sqrt{a b}(a+b)^{2}\left(\Pi_{c}+\Pi_{s}\right) H_{1}}{6\left(1-y^{2}\right) s(y)^{2}}, \\
\gamma_{y \chi}^{(1)} & =\frac{(a-b)(a+b)^{2} y\left(1-y^{2}\right) H_{4}}{2 s(y)^{2}}, \quad \gamma_{y \sigma}^{(1)}=\frac{(a-b)(a+b)^{2} y^{2} \tilde{H}_{4}}{2 s(y)^{2}}, \\
\gamma_{\chi \chi}^{(1)} & =-\frac{\sqrt{a b}(a+b)^{2}\left(\Pi_{c}+\Pi_{s}\right)\left[2 H_{1} H_{2}-3 H_{3} s(y)^{3}\right]\left(1-y^{2}\right)}{6 s(y)^{5}}, \\
\gamma_{\sigma \sigma}^{(1)} & =-\frac{\sqrt{a b}(a+b)^{2}\left(\Pi_{c}+\Pi_{s}\right)\left[2 H_{1} \tilde{H}_{2}-3 \tilde{H}_{3} s(y)^{3}\right] y^{2}}{6 s(y)^{5}}, \\
\gamma_{\chi \sigma}^{(1)} & =-\frac{\sqrt{a b}(a+b)^{2}\left(\Pi_{c}+\Pi_{s}\right)\left[2 H_{0} H_{1}-3 a b s(y)^{3}\right] y^{2}\left(1-y^{2}\right)}{6 s(y)^{5}} .
\end{aligned}
$$

Here, the functions $H_{a}$ and $\tilde{H}_{a}$ are given by

$$
\begin{aligned}
H_{0}= & (a+b)^{3} \Pi_{c} \Pi_{s}+a b^{2}\left[1+\alpha-\left(\Pi_{c}+\Pi_{s}\right)^{2}+\left(1-y^{2}\right)\right]+a^{2} b\left[1+\alpha-\left(\Pi_{c}+\Pi_{s}\right)^{2}+y^{2}\right], \\
H_{1}= & (a+b)^{2}\left(1-\Pi_{c}^{2}+\Pi_{s}^{2}\right)-3(a-b)^{2} y^{2}\left(1-y^{2}\right)+\left[a^{2}(3+\alpha)-\alpha b^{2}\right] y^{2}+\left[b^{2}(3+\alpha)-\alpha a^{2}\right]\left(1-y^{2}\right), \\
H_{2}= & a b(a+b)\left(\Pi_{c}+\Pi_{s}\right)^{2}+\left(a^{3} \Pi_{c}^{2}+b^{3} \Pi_{s}^{2}\right) y^{2} \\
& -a b\left[a\left(1+\alpha-\Pi_{c}^{2}+2 \Pi_{c} \Pi_{s}\right)+b\left(2+\alpha-\Pi_{s}^{2}+2 \Pi_{c} \Pi_{s}\right)\right] y^{2}-a b(a-b) y^{4}, \\
H_{3}= & (a+b)^{2}\left(\Pi_{c}^{2}-\Pi_{s}^{2}\right)+\left[a^{2}(1+\alpha)-b^{2}(2+\alpha)\right] y^{2}+(a-b) y^{4}, \\
H_{4}= & a b\left(\Pi_{c}+\Pi_{s}\right)+(a-b)\left(a \Pi_{c}-b \Pi_{s}\right) y^{2}, \\
H_{5}= & (a+b)\left(\Pi_{c}+\Pi_{s}\right)\left(b \Pi_{c}-a \Pi_{s}\right)+(a-b)[a(1+\alpha)+b(2+\alpha)] y^{2}+(a-b)^{2} y^{4},
\end{aligned}
$$

with $\left(\tilde{H}_{2}, \tilde{H}_{3}, \tilde{H}_{4}, \tilde{H}_{5}\right)$ being obtained from $\left(H_{2}, H_{3}, H_{4}, H_{5}\right)$ by making the replacements

$$
\tilde{H}_{a}=\left.H_{a}\right|_{\left(y \rightarrow \sqrt{1-y^{2}}, a \rightarrow b, b \rightarrow a\right)} .
$$

( $H_{0}$ and $H_{1}$ are invariant, or "self-dual," under this transformation.) Note that, as in the analogous discussion in the case of the 4-charge black holes in four dimensions, our choice of coordinate redefinition in (4.11) with $u=-\frac{1}{8}$ has ensured that $h_{0}^{y}=0$, which is essential for the existence of a conserved Aretakis charge.

From the expressions in (4.14), we find that

$$
\left.\frac{\partial \log \gamma}{\partial \rho}\right|_{\rho=0}=\frac{(a+b)^{2}\left(\Pi_{c}-\Pi_{s}\right)}{2 \sqrt{a b}}
$$

for the five-dimensional 3-charge rotating extremal black holes, and $\sqrt{\bar{\gamma}}=\sqrt{a b}(a+b)^{2}\left(\Pi_{c}+\Pi_{s}\right) y$, so the Aretakis charge (2.5) is given by

$$
\begin{aligned}
Q_{A}= & \sqrt{a b}(a+b)^{2}\left(\Pi_{c}+\Pi_{s}\right) \int y d y d \chi d \sigma \\
& \times\left[2 \psi_{1}+\frac{(a+b)^{2}\left(\Pi_{c}-\Pi_{s}\right)}{4 \sqrt{a b}} \psi_{0}\right] .
\end{aligned}
$$

\section{INVERSION AND NEWMAN-PENROSE CHARGES}

\section{A. Inversion and weakly asymptotically flat spacetimes in four dimensions}

Conserved Aretakis charges are defined on the horizon of an extremal black hole. A different kind of conserved charge, known as a Newman-Penrose (NP) charge, is defined at null infinity in an asymptotically flat spacetime. It was shown in [11] that in the case of an extremal Reissner-Nordström black hole, there exists an inversion symmetry, which takes the form $\rho \rightarrow 1 / r$, where $\rho$ is the radial coordinate of the ERN black hole written in Gaussian 
null coordinates, under which the inverted metric is conformally related to the ERN metric again. This symmetry was employed in $[9,10]$ in order to show that the Aretakis charge for a massless scalar field in the ERN background was related to the Newman-Penrose charge for the massless scalar, calculated at future null infinity in the same ERN metric.

The fact that the conformal inversion of the extremal Reissner-Nordström black hole gives back precisely the same ERN metric is of itself inessential for the purpose of mapping the Aretakis charge into a Newman-Penrose charge in the conformally inverted metric. In [12], a general discussion was given in which an extremal black hole metric, written in Gaussian null coordinates as in (2.1), was conformally inverted to give a metric that was weakly asymptotically flat. Under appropriate conditions, the Aretakis charge in the original extremal black hole metric can be mapped into a Newman-Penrose charge in the weakly asymptotically flat metric. The conformal inversion is effected by starting from (2.1) and then taking

$$
\rho=\frac{1}{r}, \quad v=u, \quad d s^{2}=\frac{L(x)^{2}}{r^{2}} d \tilde{s}^{2},
$$

and the weakly asymptotically flat metric is thus given by [12]

$$
\begin{aligned}
d \tilde{s}^{2}= & -F e^{2 \beta} d u^{2}-2 e^{2 \beta} d u d r \\
& +r^{2} h_{I J}\left(d x^{I}-C^{I} d u\right)\left(d x^{J}-C^{J} d u\right),
\end{aligned}
$$

with

$$
\begin{gathered}
\beta=0, \quad C^{I}=\frac{h^{I}}{r}=h_{0}^{I} r^{-1}+h_{1}^{I} r^{-2}+\cdots, \\
h_{I J}=L(x)^{-2} \gamma_{I J}=L(x)^{-2}\left(\bar{\gamma}_{I J}+\gamma_{I J}^{(1)} r^{-1}+\cdots\right) .
\end{gathered}
$$

The appellation "weakly asymptotically flat" signifies the fact that the usual definition of asymptotic flatness has been weakened in two respects. First, the vector $C^{I}$ is allowed to have terms at order $1 / r$ in its asymptotic expansion, in contrast to the usual requirement of leading $1 / r^{2}$ falloff for asymptotic flatness. Second, the leading-order metric on the $r=$ constant spatial sections is not restricted to being that of a round sphere.

In the case of static spherically symmetric extremal black hole metrics in four dimensions, the distinction between weakly asymptotically flat and asymptotically flat in the conformally inverted metric is irrelevant, since $h^{I}=0$ and hence $C^{I}=0$, and in addition $L(x)=$ constant and $L(x)^{-2} \bar{\gamma}_{I J}$ is just the metric on the unit round sphere, implying that $h_{I J}$ at leading order is also the round sphere metric. In [12] the conformal inversion for static spherically symmetric extremal black holes was employed in order to relate the Aretakis and Newman-Penrose charges in some more general examples, such as the 4-charge extremal static black holes of four-dimensional STU supergravity.

A crucial point about the conformal inversion in the static four-dimensional extremal black holes considered in [12] is that the Ricci scalar is either zero (as in the extremal Reissner-Nordström example) or else it goes to zero sufficiently rapidly (as in the general 4-charge STU examples) that in both the Aretakis and the NewmanPenrose calculations, one can as well replace the massless scalar operator $\square$ by the conformally invariant operator $\square-\frac{1}{6} R$. This means that one can invoke the consequent conformal relation between the solutions of the scalar operator in the original and the conformally inverted metrics, in order to establish a mapping between the Aretakis and the Newman-Penrose charges.

If the extremal black hole is stationary but not static, the mapping between the Aretakis charge in the black hole metric and the Newman-Penrose charge in the conformally inverted metric with $\rho \rightarrow 1 / r$ will break down. Even if we consider the simplest example, namely the extremal Kerr metric, the conformal mapping between the solutions of the scalar wave equation will fail. There is no problem with the calculation for the Aretakis charge in the extremal Kerr metric, since the Ricci scalar vanishes and there is no difference between the massless scalar operator $\square$ and the conformally invariant operator $\square-\frac{1}{6} R$. However, in the weakly asymptotically flat metric $d \tilde{s}^{2}$ obtained by conformal inversion, the Ricci scalar is nonvanishing and it has a $1 / r^{2}$ falloff at large $r$. Thus one can straightforwardly see from the form of the four-dimensional extremal rotating 4-charge metrics in Gaussian null coordinates obtained in Sec. III that after turning off the charges to give the Kerr metric, and making the inversion and conformal scaling

$$
\rho=\frac{1}{r}, \quad d \tilde{s}^{2}=\frac{r^{2}}{L(x)^{2}} d s^{2},
$$

the Ricci scalar $\tilde{R}$ calculated in the conformally rescaled metric has the leading-order form

$$
\tilde{R}=\frac{6\left(1-5 y^{2}\right)}{\left(1+y^{2}\right)^{2}} \frac{1}{r^{2}}+\mathcal{O}\left(\frac{1}{r^{3}}\right) .
$$

When one looks at the calculation of the Newman-Penrose charge (which we shall discuss in detail below), one finds that while there does exist a Newman-Penrose charge for a massless scalar obeying $\tilde{\square} \tilde{\psi}=0$ in the WAF metric, there does not exist a Newman-Penrose charge for a scalar obeying the conformally invariant equation $\left(\tilde{\square}-\frac{1}{6} \tilde{R}\right) \tilde{\psi}=0$. The problem is that the Ricci scalar with its $1 / r^{2}$ falloff gives an obstruction to the existence of a conserved charge. There is therefore no conformal mapping that allows one to relate the Aretakis charge for a scalar obeying $\square \psi=0$ in 
the extremal Kerr metric to the Newman-Penrose charge for a scalar obeying $\tilde{\square} \tilde{\psi}=0$ in the related WAF metric. This difficulty can be seen in a calculation of the Aretakis and the Newman-Penrose charges for this example that was carried out in [13].

It is useful nevertheless to examine in detail the construction of the Newman-Penrose charge for a massless scalar field in a general weakly asymptotically flat spacetime, and we shall now present the calculation in a general spacetime dimension $n$.

\section{B. Inversion and WAF spacetimes in higher dimensions}

In dimensions greater than four, further complications can arise. If we consider even a static extremal black hole, such as a higher-dimensional extremal Reissner-Nordström metric, the Ricci scalar no longer vanishes (since only in four dimensions is the electromagnetic energy-momentum tensor trace-free), and in fact it approaches a constant on the horizon. For example, writing the five-dimensional ERN metric in the Gaussian null form (2.1), it is given by

$$
\begin{aligned}
L(x)^{2} & =\frac{Q}{4}, \quad F=\rho^{2}\left(1+\frac{\sqrt{Q}}{8} \rho\right)^{2}\left(1+\frac{\sqrt{Q}}{4} \rho\right)^{-4}, \\
\gamma_{I J} & =Q\left(1+\frac{\sqrt{Q}}{4} \rho\right)^{2} \omega_{I J}
\end{aligned}
$$

where $Q$ is the electric charge and $\omega_{I J}$ is the metric on the unit 3-sphere. The Ricci scalar is given by

$$
R=-\frac{2}{Q}\left(1+\frac{\sqrt{Q}}{4} \rho\right)^{-6},
$$

which approaches the constant $-2 / Q$ on the horizon at $\rho=0$. This implies that although there exists an Aretakis charge for a scalar field obeying the massless wave equation $\square \psi=0$, there will be no conserved charge for a scalar obeying the conformally invariant wave equation, which is $\left(\square-\frac{3}{16} R\right) \psi=0$ in five dimensions.

Furthermore, after the inversion with $\rho \rightarrow 1 / r$ and $v \rightarrow u$, and the conformal scaling to $d \tilde{s}_{5}^{2}=\left(r^{2} / L(x)^{2}\right) d s_{5}^{2}$, we obtain the WAF metric

$$
d \tilde{s}_{5}^{2}=-\frac{\left(1+\frac{\sqrt{Q}}{8 r}\right)^{2}}{\left(1+\frac{\sqrt{Q}}{4 r}\right)^{4}} d u^{2}-2 d u d r+4 r^{2}\left(1+\frac{\sqrt{Q}}{4 r}\right)^{2} d \Omega_{3}^{2},
$$

where $d \Omega_{3}^{2}$ is the metric on the unit 3-sphere. This has a Ricci scalar that falls off only as $r^{-2}$ at infinity, and in fact

$$
\tilde{R}=-\frac{9}{2 r^{2}}+\mathcal{O}\left(\frac{1}{r^{3}}\right)
$$

The reason why this happens in higher dimensions but not in four is that now, in the conformally inverted WAF metric, the metric on the $r=$ constant surfaces is not simply approaching $r^{2} d \Omega^{2}$, but instead a nonunit constant times $r^{2} d \Omega^{2}$. [The constant is 4 in the five-dimensional example in (5.8).] This means the spatial metric is not locally approaching the Euclidean metric at large $r$, and this is responsible for the slower falloff of the Ricci scalar.

As discussed previously, a Ricci scalar with this falloff contributes in the calculation of Newman-Penrose charges if one considers the conformally invariant scalar wave operator.

As we shall see in the next section, there is actually a further complication in dimensions greater than four, when one attempts to construct conserved Newman-Penrose charges at null infinity.

\section{Newman-Penrose charges in four and higher dimensions}

In this section, which is concerned exclusively with the calculation of Newman-Penrose charges, we shall drop the tildes that we were previously using to denote the weakly asymptotically flat metric. The general solution of $\square \Psi=0$ in the weakly asymptotically flat metric (5.2) in $n$ dimensions has a large- $r$ expansion of the form

$$
\begin{aligned}
\Psi(r, u, x) & =\Psi_{0}(u, x) r^{-\gamma}+\Psi_{1}(u, x) r^{-\gamma-1}+\cdots, \\
\gamma & =\frac{n-2}{2} .
\end{aligned}
$$

If the $(n-2)$-dimensional metric $h_{I J}$ in the WAF metric (5.2) is expanded as

$$
h_{I J}(r, u, x)=\omega_{I J}+h_{I J}^{(1)} r^{-1}+\cdots,
$$

we may choose a coordinate gauge where $\sqrt{h}=\zeta(r) \sqrt{\omega}$, with

$$
\zeta(r)=1+\zeta_{0} r^{-1}+\zeta_{1} r^{-2}+\cdots .
$$

Substituting (5.10) into $\square \Psi=0$, evaluated in the WAF metric background, we find that at the leading order in the large- $r$ expansion,

$$
\begin{aligned}
& \frac{\partial}{\partial u}\left[2 \Psi_{1}+\zeta_{0} \Psi_{0}\right]-\frac{(n-2)(n-4)}{4} \Psi_{0}-\frac{n-4}{2} C_{0}^{I} \partial_{I} \Psi_{0} \\
& +\frac{n-2}{2} D_{I}\left(C_{0}^{I} \Psi_{0}\right)+D^{I} D_{I} \Psi_{0}=0
\end{aligned}
$$

where $C^{I}$ has an expansion of the form $C^{I}=C_{0}^{I} r^{-1}+$ $C_{1}^{I} r^{-2}+\cdots$, and where $D_{I}$ denotes the covariant derivative in the $\omega_{I J}$ metric.

In $n=4$ dimensions, we can obtain a conserved charge by integrating (5.13) over the 2 -sphere with metric $\omega_{I J}$ : 


$$
Q_{\mathrm{NP}}=\int \sqrt{\omega} d^{2} x\left[2 \Psi_{1}+\zeta_{0} \Psi_{0}\right]
$$

In a general dimension $n \neq 4$ there are two obstructions to obtaining a conserved charge. First, the term $\frac{n-4}{2} C_{0}^{I} \partial_{I} \Psi_{0}$ is not a total derivative in general. Note, however, that if $C_{0}^{I}$ vanishes when $I$ lies in the direction(s) associated with latitude type coordinates on the sphere (i.e., directions that are not associated with Killing vectors), then this term can be rewritten as the total derivative $\frac{n-4}{2} D_{I}\left(C_{0}^{I} \Psi_{0}\right)$, since the remaining, azimuthal, sphere coordinates are associated with Killing directions. As we saw earlier, $C_{0}^{I}$ will indeed vanish in the nonazimuthal directions in the case of the WAF metrics obtained by conformal inversion of extremal black hole metrics, since $h_{0}^{I}=0$ in those directions in the black hole metrics. This still leaves the problem of the term $-\frac{(n-2)(n-4)}{4} \Psi_{0}$ in (5.13). This term implies that there can be no Newman-Penrose charge for a massless scalar obeying $\square \Psi=0$ in any dimension higher than $n=4$.

It is perhaps worth remarking that in the case of a WAF metric obtained by conformal inversion of a static spherically symmetric extremal black hole in $n \geq 5$ dimensions, the Ricci scalar goes like $1 / r^{2}$ at large distance and thus it would make a contribution in the NP charge calculation at the leading order in a large- $r$ expansion if one were to add an $R \Psi$ term to the massless wave equation $\square \Psi=0$. If the coefficient of this term were chosen appropriately, it could be arranged to cancel the term $-\frac{(n-2)(n-4)}{4} \Psi_{0}$ in (5.13), thus allowing the existence of a conserved NP charge. [See Eq. (5.9) for the calculation of the Ricci scalar term for the conformal inversion of the five-dimensional extremal Reissner-Nordström metric.] However, as may be readily checked, the coefficient of $R \Psi$ that would be needed to achieve this cancellation appears to have no other related significance. In particular, it is not equal to the coefficient that would be needed for the conformally invariant scalar operator.

\section{CONCLUSIONS}

In this paper, we have constructed the metrics in Gaussian null coordinates for certain classes of extremal rotating black holes in supergravity theories, as expansions in the radial coordinate at a sufficient order to be able to calculate the conserved Aretakis charges on the horizon. Specifically, we did this for the extremal rotating black holes in four-dimensional STU supergravity that carry four independent electric charges (with the special case of the Kerr-Newman black hole when the four charges are equal), and also for the general extremal rotating 3-charge black holes in five-dimensional STU supergravity. We then obtained the explicit expressions for the simplest of the Aretakis charges for a massless scalar field in each case.
We also investigated the possibility of relating the Aretakis charge on the horizon of the extremal black hole to the Newman-Penrose charge at $\mathcal{I}^{+}$in the metric obtained by performing an inversion of the radial coordinate, after the extraction of an appropriate conformal factor. This relation was studied for four-dimensional spacetimes in [12], where various examples of the mapping were obtained for classes of static extremal black holes. In the present paper we showed that such a mapping becomes problematical for extremal rotating black holes, because after conformal inversion the resulting weakly asymptotically flat metric has a Ricci scalar whose falloff at large $r$ is sufficiently slow that one cannot treat the massless scalar equation $\square \psi=0$ as being equivalent to the conformally invariant equation $\left(\square-\frac{1}{6} R\right) \psi=0$ for the purpose of calculating the Newman-Penrose charge. This means that the ability to relate the solutions for the scalar field in the original extremal metric and in the conformally inverted metric is lost in the case of rotating black holes, at least if we consider just a simple $\rho \rightarrow 1 / r$ inversion. In turn, one cannot by this means relate the Aretakis and NewmanPenrose charges for extremal rotating four-dimensional black holes.

As we then discussed, the situation becomes worse in dimensions $n>4$. The extremal black holes (static or rotating) still admit conserved Aretakis charges for a massless scalar field, but there are no Newman-Penrose charges for a massless scalar in any asymptotically flat spacetime of dimension $n>4$. Thus it appears that the mapping between Aretakis and Newman-Penrose charges is exclusively a four-dimensional phenomenon.

There remain a number of directions for further study. First, it would be of interest to generalize the construction of the extremal rotating four-dimensional STU black holes in Gaussian null coordinates to the general case of eight charge parameters (independent electric and magnetic charges carried by each of the four gauge fields; the solution for the 8-charge rotating black holes is given in [17]). It would also be of interest to study the analogous conserved Aretakis and Newman-Penrose charges for higher-spin fields in the charged supergravity black hole backgrounds. Examples would include Maxwell fields, and also perturbations of the background metrics themselves.

\section{ACKNOWLEDGMENTS}

We are grateful to Hadi Godazgar, Mahdi Godazgar, Carmen Li, and James Lucietti for helpful discussions. The work of M. C. is supported in part by the DOE (HEP) Award No. DE-SC0013528, the Fay R. and Eugene L. Langberg Endowed Chair (M. C.) and the Slovenian Research Agency (ARRS No. P1-0306). The work of C. N.P. is supported in part by DOE Grant No. DEFG02-13ER42020. 
[1] S. Aretakis, Stability and instability of extreme ReissnerNordström black hole spacetimes for linear scalar perturbations I, Commun. Math. Phys. 307, 17 (2011).

[2] S. Aretakis, Stability and instability of extreme ReissnerNordström black hole spacetimes for linear scalar perturbations II, Ann. Inst. Henri Poincaré 12, 1491 (2011).

[3] S. Aretakis, Horizon instability of extremal black holes, Adv. Theor. Math. Phys. 19, 507 (2015).

[4] S. Aretakis, A note on instabilities of extremal black holes under scalar perturbations from afar, Classical Quantum Gravity 30, 095010 (2013).

[5] J. Lucietti and H.S. Reall, Gravitational instability of an extreme Kerr black hole, Phys. Rev. D 86, 104030 (2012).

[6] H. K. Kunduri, J. Lucietti, and H. S. Reall, Near-horizon symmetries of extremal black holes, Classical Quantum Gravity 24, 4169 (2007).

[7] C. Li and J. Lucietti, Transverse deformations of extreme horizons, Classical Quantum Gravity 33, 075015 (2016).

[8] C. Li, Extreme black holes and near-horizon geometries, Ph.D. thesis, University of Edinburgh, 2015.

[9] P. Bizon and H. Friedrich, A remark about wave equations on the extreme Reissner-Nordström black hole exterior, Classical Quantum Gravity 30, 065001 (2013).
[10] J. Lucietti, K. Murata, H. S. Reall, and N. Tanahashi, On the horizon instability of an extreme Reissner-Nordström black hole, J. High Energy Phys. 03 (2013) 035.

[11] W.E. Couch and R. J. Torrence, Conformal invariance under spatial inversion of extreme Riessner-Nordström black holes, Gen. Relativ. Gravit. 16, 789 (1984).

[12] H. Godazgar, M. Godazgar, and C. N. Pope, Aretakis charges and asymptotic null infinity, Phys. Rev. D 96, 084055 (2017).

[13] M. Cvetič and A. Satz, General relation between Aretakis charge and Newman-Penrose charge, Phys. Rev. D 98, 124035 (2018).

[14] M. Cvetič and D. Youm, Entropy of nonextreme charged rotating black holes in string theory, Phys. Rev. D 54, 2612 (1996).

[15] Z.-W. Chong, M. Cvetič, H. Lü, and C. N. Pope, Charged rotating black holes in four-dimensional gauged and ungauged supergravities, Nucl. Phys. B717, 246 (2005).

[16] M. Cvetič and D. Youm, General rotating five-dimensional black holes of toroidally compactified heterotic string, Nucl. Phys. B476, 118 (1996).

[17] D. D. K. Chow and G. Compère, Black holes in $N=8$ supergravity from $S O(4,4)$ hidden symmetries, Phys. Rev. D 90, 025029 (2014). 